Results 23 patients, from two hospitals, received the pilot intervention. 31 interviews were completed. Patients were generally positive about the assessment, openly discussing concerns and coping strategies in all domains. Professionals considered that the intervention was feasible and potentially valuable. Positive actions included facilitating discussion with their GP on palliative use of opiates, reinforcing referral to pulmonary rehabilitation and providing contact details of local voluntary services. However, fewer unmet needs were identified than expected, and some patients were reluctant to access proffered services/information. Participants highlighted overlap with discharge planning and case management provided by community teams.

Conclusion The HELP COPD holistic assessment was feasible and generally welcomed by patients. Fewer actions were identified than anticipated; perhaps due to a combination of inappropriate timing, reluctance to accept social services, duplication by existing services and long-term adaptation reducing recognition of need and of the seriousness of the condition.

Conflict of Interest and Funding This work was supported by The Dunhill Medical Trust [grant number: R210/0711]. No conflicts of interest to declare.

\title{
HELPING PEOPLE WITH VERY SEVERE COPD: FEASIBILITY STUDY OF A NOVEL HOLISTIC INTERVENTION
}

Marilyn Kendall, ${ }^{1}$ Susan Buckingham, ${ }^{1}$ Susie Ferguson, ${ }^{1,2}$ Aziz Sheikh, ${ }^{1}$ Scott Murray, ${ }^{1}$ William MacNee, ${ }^{2}$ Patrick White, ${ }^{3}$ Allison Worth, ${ }^{4}$ Kirsty Boyd, ${ }^{1}$ Hilary Pinnock'. 'University of Edinburgh, Edinburgh, UK; ${ }^{2}$ Centre for Inflammation Research, University of Edinburgh; ${ }^{3}$ Department of Primary Care and Public Health Sciences, Kings College London; ${ }^{4}$ Wellcome Trust Clinical Research Facility, University of Edinburgh

\subsection{6/bmjspcare-2014-000654.23}

Background Chronic Obstructive Pulmonary Disease (COPD) is the third leading cause of death worldwide. Research shows that people with severe COPD have unmet needs and that the traditional palliative care model is not appropriate.

Aim To pilot and evaluate the HELP-COPD intervention-a proactive holistic assessment of supportive and palliative care needs. Method The intervention comprised a home visit by a respiratory nurse, following discharge from hospital after an exacerbation, to discuss social/psychological/spiritual/physical needs, provide advice/information and make further clinical/social care referrals as appropriate. A HELP-COPD Action Plan was completed and a summary given to the patient and their general practitioner. Follow-up included three phone calls over six months.

Qualitative evaluation comprised interviews with participating patients, lay-carers and health professionals, alongside documentary analysis of the completed action plans. Interviews were recorded, transcribed and entered into NVivo for thematic analysis. 